\title{
Third and Fifth Order IMDs at Carrier Position
}

\author{
Mohamad Kadim Suaidi ${ }^{1}$, Lisa Yong ${ }^{2}$ and Awangku Abdul Rahman B. Pgn. Hj. Yusof ${ }^{3}$ \\ ${ }^{1}$ University-Industry Centre (UNIC), Kolej Universiti Teknikal Kebangsaan Malaysia, Ayer Keroh, 75450 Melaka, Malaysia \\ ${ }^{2}$ Engineering Faculty, Universiti Malaysia Sarawak, Kota Samarahan, Sarawak, Malaysia. \\ ${ }^{3}$ Research and Innovation Management Centre (RIMC), Universiti Malaysia Sarawak, 94300 Kota Samarahan, Sarawak, Malaysia. \\ kadim@kutkm.edu.my, ylisa@feng.unimas.my, pyaarahman@rimc.unimas.my
}

\begin{abstract}
The third, instead of the fifth order intermodulation distortion $\left(\mathrm{IMD}_{3}\right)$, is often thought to limit the radio-over-fibre system performance as it tends to fall in-band. The Golomb Ruler based frequency plan can overcome this $\mathrm{IMD}_{3}$ problem. However, its effect on the $\mathrm{IMD}_{5}$ is not known. A laser model was derived based on Volterra Series with electrical parasitics to simulate the carrier position $\mathrm{IMD}_{3}$ and $\mathrm{IMD}_{5}$ for the Golomb Ruler and equally spaced frequency plans at low and high RF input level, supported by low bias current. The $\mathrm{IMD}_{5}$ was more dominant than the $\mathrm{IMD}_{3}$ for the equally spaced frequency plan at high RF input level. The opposite was observed at low RF input. The Golomb Ruler based frequency plan led to no $\mathrm{IMD}_{3} \mathrm{~s}$ but low levels of $\mathrm{IMD}_{5}$ were still present the carrier position but they were lower than those due to the equal frequency spaced channels.
\end{abstract}

Keywords: Golomb Ruler, third and fifth intermodulation distortions

\section{Introduction}

The radio-over-fibre system ( $\mathrm{ROF}$ ) is used to transport fixed or mobile wireless signals by employing the optical fibre. The wide dynamic range of the wireless environment must be met by the optical portion of the ROF system, especially in the uplink as the wireless signal intercepted from the cell edge can be very weak or it can be very strong when it is close to the based station. The dynamic range can be limit by the third order intermodulation distortions $\left(\mathrm{IMD}_{3}\right)$ of type $2 f_{1}-f_{2}$ and $f_{1}+f_{2}-f_{3}$ as they are liable to fall inband for narrowband systems [1]. The electric-tooptical (E/O) conversion stage in the ROF is one of the contributors to this distortion [2]. The $\mathrm{IMD}_{3}$ can be reduced by rearranging the channels to be used according the frequency plans in [3] and [4]. As the mobile communication system has to follow certain frequency management or channel assignment scheme and is subjected to interference issues such as cochannel interference [5], the frequency plan being exercised cannot be simply altered to reduce the $\mathrm{IMD}_{3}$ generated by the E/O conversion. The signal extraction with frequency arrangement (SEFA) had been recommended as means to improve the dynamic range by reducing the $\mathrm{IMD}_{3}$ through reassigning the received signals after intercepting them [2], [6]. In addition, the signal level compression (SLC) scheme that would boost the levels of weak signals was also suggested to improve the dynamic range [2], [7]. A frequency plan is needed for the SEFA scheme to be implemented. Hunziker suggested arranging the channels to be used in an unequal manner by employing the Golomb Ruler [1] as it avoids $\mathrm{IMD}_{3}$ from appearing at the these channels. The Golomb Ruler is a set of non-negative integers or marks such that the distance between any pair of marks are unique and it begins with ' 0 ' [8], [9]. The Optimum Golomb Ruler (OGR) is a Golomb Ruler that gives the shortest length for a given number of marks [9]. The OGR for five marks is indicated in Figure 1.

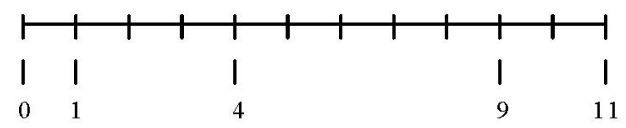

Figure 1: The Golomb Ruler for five marks.

Though the Golomb Ruler based frequency plan can lead to zero $\mathrm{IMD}_{3}$ at carrier position, other intermodulation distortions might be present at the carrier positions. Therefore this paper aims at presenting a ROF system laser model derived using Volterra Series and compute the $\mathrm{IMD}_{3}$ and fifth orders intermodulation distortions ( $\mathrm{IMD}_{5}$ ) at carrier positions for channels in the Golomb Ruler frequency plan and equal frequency spacing. The $\mathrm{IMD}_{5}$ is looked into as it is the next odd order distortion after the $\mathrm{IMD}_{3}$. Individual $\mathrm{IMD}_{5} \mathrm{~S}$ might be insignificant as compared to $\mathrm{IMD}_{3} \mathrm{~s}$ but their levels might escalate if their quantities increase and the input magnitude intensifies.

\section{Volterra Series Laser Model}

Volterra Series is used to model weakly nonlinear systems with memory. The laser diode output $s(t)$ is expressed as (1) in Volterra Series [10]:

$$
s(\mathrm{t})=\sum_{n=1}^{\infty} \int_{-\infty}^{\infty} \ldots \int_{-\infty}^{\infty} \mathrm{h}_{n}\left(\mathrm{u}_{1} \ldots u_{n}\right) \cdot \prod_{l=1}^{n} i\left(\mathrm{t}-\mathrm{u}_{l}\right) \mathrm{du}_{l} \ldots d u_{n}
$$

